\title{
3. ODP LEG 104, UNDERWAY GEOPHYSICS ${ }^{1}$
}

\author{
Shipboard Scientific Party ${ }^{2}$
}

\section{INTRODUCTION}

We obtained geophysical site surveys during Leg 104 of the Ocean Drilling Program between Bremerhaven, Federal Republic of Germany and St. John's, Newfoundland, Canada. Five lines of geophysical data were collected on Leg 104. Line 1 data were collected before the beacon drop at Site 642; Line 2 when underway between Sites 642 and 643; Line 3 between Sites 643 and 644; Line 4 during the transit from Site 644 to Stavanger, Norway; and Line 5 between Stavanger and St. John's (Leg 104 Part B). We were underway 13.9 days $(21 \%$ percent of the time) during the 65.4 days of Leg 104 and traveled a total of 3,087 miles $(4980 \mathrm{~km})$.

The onboard instrumentation included two precision echosounders, a magnetometer, seismic-reflection profilers, and a satellite-navigation system. The instruments were maintained and operated by the ODP marine technicians in cooperation with the scientific party and the officers and crew of SEDCO-FOREX, Inc.

\section{NAVIGATION}

Navigation data were collected on the ship's bridge by a Decca navigation system and a Magnavox MX702A satellite-navigation system (SATNAV). Positions were obtained with these systems through the entire 57 days spent at sea (Table 1). The general ship track chart for Leg 104 is shown in Figure 1. Detailed navigation of the ship surveys for site location, reference multichannel seismic lines, and the positions of Leg 104 drill sites are illustrated in Figure 2.

\section{BATHYMETRIC DATA RECORDING}

Bathymetric data were obtained with both $3.5-\mathrm{kHz}$ and 12 $\mathrm{kHz}$ echo-sounders using a Raytheon recorder system for the $3.5-\mathrm{kHz}$ and an EDO $248 \mathrm{C}$ recorder for the $12-\mathrm{kHz}$ instrument. Unfortunately, because of transducer location, the quality of the recorded data is very poor at times when the ship operated

${ }^{1}$ Eldholm, O., Thiede, J., Taylor, E., et al., 1987. Proc., Init. Repts. (Pt. A), $O D P, 104$.

2 Olav Eldholm, (Co-Chief Scientist), University of Oslo, Oslo, Norway; Jörn Thiede (Co-Chief Scientist), Christian-Albrechts-Universität, Kiel, FRG; Elliott Taylor (Staff Scientist), ODP, Texas A\&M University, College Station, TX; Colleen Barton, Stanford University, Stanford, CA; Kjell Bjorklund, University of Oslo, Oslo, Norway; Ulrich Bleil, Universität Bremen, Bremen, FRG; Paul R. Ciesielski, University of Florida, Gainesville, FL; Alain Desprairies, Université de Paris Sud, Orsay, France; Diane Donnally, Amoco Production Co., New Orleans, LA; Claude Froget, Faculté des Sciences de Luminy, Marseilles, France; Robert Goll, IKU/SINTEF, Trondheim, Norway; Rudiger Henrich, Christian-AlbrechtsUniversität, Kiel, FRG; Eystein Jansen, University of Bergen, Bergen, Norway; Larry Krissek, Ohio State University, Columbus, OH; Keith Kvenvolden, USGS, Menlo Park, CA; Anne LeHuray, Lamont-Doherty Geological Observatory, Palisades, NY; David Love, University of Waterloo, Ontario, Canada; Peter Lysne, Sandia National Laboratory, Albuquerque, NM; Thomas McDonald, Texas A\&M University, College Station, TX; Peta Mudie, Geological Survey of Canada, Nova Scotia, Canada; Lisa Osterman, Smithsonian Institution, Washington, DC; Lindsay Parson, Institute of Oceanographic Sciences, Surrey, UK; Joseph Phillips, University of Texas, Austin, TX; Alan Pittenger, Texas A\&M University, College Station, TX; Gunnbjørg Qvale, Norsk Hydro, Oslo, Norway; Günther Schönharting, University of Copenhagen, Copenhagen, Denmark; Lothar Viereck, Ruhr Universität, Bochum, FRG. at speeds over $6 \mathrm{kt}$. A total of 2,020 n. mi. (3258 km) of bathymetric coverage was collected on Leg 104 and is displayed in Figures 3 and 4 . The real-time recording parameters of the data are provided in Table 2 .

\section{MAGNETICS}

A Geometrics 801 proton precession magnetometer was towed between sites and along the transits from Site 644 to Stavanger and from Stavanger to St. John's. The analog record is incomplete; however, summaries of the results are shown in Figures 3 and 4 . Preliminary studies of the magnetic record suggest that some anomalies may be detected in future data processing.

\section{SEISMIC-REFLECTION PROFILES}

Seismic-reflection profiles were collected over 2,367 n. mi. (3818 km) during Leg 104 Parts A and B (Fig. 3). The seismicreflection coverage is shown by means of the bar in Figures 3 and 4 , and records are available from the Data Base Supervisor, Ocean Drilling Program. These data were recorded with the equipment described here.

\section{Sources}

The seismic sources used aboard the JOIDES Resolution during Leg 104 were usually two 80 -in. ${ }^{3}$ waterguns. Several seismic sources were used during the transit to St. John's: two 80 -in. ${ }^{3}$ guns were deployed the first 3 days of the transit. Later, one of the waterguns was replaced by an airgun for a few hours. The transit ended with one watergun as the seismic source.

\section{Streamer-hydrophones}

One Teledyne streamer was deployed at the fantail. The 100m-long streamer contains sixty active sections and was towed approximately $500 \mathrm{~m}$ behind the vessel. The towing depth was set by external depth depressors (birds). The hydrophone elements were combined to procure a single signal.

\section{Data Recording}

The seismic system was supported by a supermicro 561 Masscomp computer as the central unit to record, process, and display the data. The Masscomp allowed data to be processed and displayed in real time on a 15 -in.-wide Printronix, a high-resolution graphic printer (160 dots per inch). The processing treatments applied are described in Table 3 . The raw data were recorded on a Cither tape, using an SEG-Y format and a density of $1600 \mathrm{bits} / \mathrm{in}$.

Seismic lines 1, 4, and 5 were displayed in the Printronix printer with the following parameters:

Traces per inch $=10$

Clip high $=0.10 \mathrm{in}$.

Clip low $=-0.10$ in.

Deflection $=0.10$ in.

Positive peaks to the right

Seismic data were also displayed in real time in analog format on two EDO 550 dry-paper recorders, using only streamers, an amplifier, and two band-pass filters (see Table 3 ). 


\section{ACKNOWLEDGMENTS}

The scientific party of Leg 104 is extremely grateful to Captain Ed Oonk and his officers and crew for their excellence in navigation and their cooperative spirit. Thanks go also to the ODP group for the many hours of watch-standing and to Mark Weiderspahn and Ali Tufayli of the University of Texas at Austin, who designed and wrote the software for the seismic digital acquisition system.

Table 1. Leg 104 navigation satellite fixes.

\begin{tabular}{|c|c|c|c|c|}
\hline $\begin{array}{c}\text { Day of } \\
\text { Year }\end{array}$ & $\begin{array}{l}\text { Time } \\
\text { UTC }\end{array}$ & $\begin{array}{l}\text { Latitude } \\
\text { N }\end{array}$ & $\begin{array}{c}\text { Longitude } \\
\text { E }\end{array}$ & Site \\
\hline 177 & 1144 & 61.68000 & 4.12000 & 642 \\
\hline 177 & 1338 & 62.06205 & 4.02408 & \\
\hline 177 & 1436 & 62.27527 & 4.00640 & \\
\hline 177 & 1550 & 62.54350 & 3.94040 & \\
\hline 177 & 1654 & 62.66670 & 3.87642 & \\
\hline 177 & 1738 & 62.93425 & 3.87250 & \\
\hline 177 & 1820 & 63.08123 & 3.83018 & \\
\hline 177 & 1842 & 63.15017 & 3.80722 & \\
\hline 177 & 1928 & 63.31750 & 3.73222 & \\
\hline 177 & 2028 & 63.52790 & 3.71772 & \\
\hline 177 & 2116 & 63.69880 & 3.67222 & \\
\hline 177 & 2304 & 64.06780 & 3.61952 & \\
\hline 177 & 2348 & 64.22552 & 3.61608 & \\
\hline 178 & 0034 & 64.38242 & 3.59470 & \\
\hline 178 & 0134 & 64.57872 & 3.58207 & \\
\hline 178 & 0408 & 65.05950 & 3.53227 & \\
\hline 178 & 0556 & 65.31185 & 3.48422 & \\
\hline 178 & 0724 & 65.51580 & 3.42270 & \\
\hline 178 & 0852 & 65.73147 & 3.34812 & \\
\hline 178 & 1158 & 66.22657 & 3.25213 & \\
\hline 178 & 1250 & 66.36618 & 3.30053 & \\
\hline 178 & 1416 & 66.59573 & 3.30407 & \\
\hline 178 & 1630 & 66.98322 & 3.04298 & \\
\hline 178 & 1710 & 67.07816 & 2.95327 & \\
\hline 178 & 1900 & 67.23337 & 2.87237 & \\
\hline 211 & 0930 & 67.22200 & 2.92933 & \\
\hline 213 & 1958 & 67.22010 & 2.93090 & 642 \\
\hline 213 & 2104 & 67.21990 & 2.93070 & \\
\hline 213 & 2146 & 67.21986 & 2.93098 & \\
\hline 214 & 0046 & 67.41513 & 2.07648 & \\
\hline 214 & 0212 & 67.55985 & 1.51277 & \\
\hline 214 & 0306 & 67.64326 & 1.26815 & \\
\hline 214 & 0548 & 67.70572 & 1.04273 & \\
\hline 214 & 0638 & 67.71385 & 1.02582 & \\
\hline 214 & 0652 & 67.71447 & 1.03243 & \\
\hline 214 & 0732 & 67.71535 & 1.03510 & \\
\hline 214 & 0752 & 67.71600 & 1.02363 & \\
\hline 219 & 0205 & 67.71535 & 1.03347 & \\
\hline 219 & 2320 & 67.71458 & 1.03683 & 643 \\
\hline 220 & 0046 & 67.64795 & 1.19952 & \\
\hline 220 & 0144 & 67.55534 & 1.67857 & \\
\hline 220 & 0532 & 67.04668 & 3.21820 & \\
\hline 220 & 0624 & 66.94047 & 3.60280 & \\
\hline 220 & 0718 & 66.83280 & 4.00718 & \\
\hline 220 & 0746 & 66.77730 & 4.21508 & \\
\hline 220 & 0816 & 66.75177 & 4.39257 & \\
\hline 220 & 0842 & 66.73238 & 4.53615 & \\
\hline 222 & 0100 & 66.67834 & 4.57667 & \\
\hline 222 & 1716 & 66.62680 & 4.53615 & 644 \\
\hline 222 & 1926 & 66.21993 & 4.55118 & \\
\hline 222 & 2022 & 66.02412 & 4.56002 & \\
\hline 222 & 2056 & 65.90332 & 4.56260 & \\
\hline 222 & 2304 & 65.44217 & 4.53017 & \\
\hline 223 & 0724 & 63.67697 & $4.37253 \mathrm{E}$ & \\
\hline 228 & 1700 & 59.50322 & $3.98238 \mathrm{~W}$ & \\
\hline 228 & 1806 & 59.48475 & 4.41725 & \\
\hline 228 & 2048 & 59.46437 & 5.56102 & \\
\hline 228 & 2158 & 59.47055 & 6.02872 & \\
\hline 228 & 2242 & 59.43044 & 6.29323 & \\
\hline 229 & 0132 & 59.36067 & 7.52420 & \\
\hline 229 & 0250 & 59.34045 & 8.08948 & \\
\hline 229 & 1012 & 59.21320 & 11.20010 & \\
\hline 229 & 1126 & 59.15897 & 11.69958 & \\
\hline 229 & 1404 & 59.09872 & 12.41238 & \\
\hline 229 & 1452 & 59.06820 & 12.75848 & \\
\hline 229 & 1554 & 59.02685 & 13.20328 & \\
\hline 229 & 1744 & 58.98457 & 13.96278 & \\
\hline 229 & 1834 & 58.97005 & 14.30970 & \\
\hline
\end{tabular}

Table 1 (continued).

\begin{tabular}{|c|c|c|c|c|}
\hline $\begin{array}{l}\text { Day of } \\
\text { Year }\end{array}$ & $\begin{array}{l}\text { Time } \\
\text { UTC }\end{array}$ & $\begin{array}{l}\text { Latitude } \\
\text { N }\end{array}$ & $\begin{array}{c}\text { Longitude } \\
\text { E }\end{array}$ & Site \\
\hline 229 & 1932 & 58.95847 & 14.69333 & \\
\hline 229 & 2022 & 58.93884 & 15.00442 & \\
\hline 229 & 2256 & 58.82787 & 15.95315 & \\
\hline 230 & 0142 & 58.70483 & 16.96750 & \\
\hline 230 & 0330 & 58.61568 & 17.59898 & \\
\hline 230 & 1032 & 58.32549 & 20.10438 & \\
\hline 230 & 1108 & 58.28738 & 20.30308 & \\
\hline 230 & 1440 & 58.08445 & 21.08597 & \\
\hline 230 & 1758 & 57.89590 & 22.19347 & \\
\hline 230 & 1856 & 57.84402 & 22.55623 & \\
\hline 230 & 1914 & 57.82398 & 22.66780 & \\
\hline 230 & 2154 & 57.69580 & 23.60088 & \\
\hline 230 & 2342 & 57.59020 & 24.20120 & \\
\hline 231 & 0130 & 57.47117 & 24.82167 & \\
\hline 231 & 0736 & 57.13935 & 26.70268 & \\
\hline 231 & 0830 & 57.07555 & 26.98342 & \\
\hline 231 & 1927 & 56.39030 & 30.27800 & \\
\hline 231 & 2038 & 56.27725 & 30.72737 & \\
\hline 231 & 2103 & 56.24854 & 30.86622 & \\
\hline 231 & 2133 & 56.20615 & 31.03930 & \\
\hline 231 & 2250 & 56.10122 & 31.44420 & \\
\hline 231 & 2308 & 56.07632 & 31.54980 & \\
\hline 232 & 0114 & 55.89147 & 32.22405 & \\
\hline 232 & 0259 & 55.73500 & 32.77150 & \\
\hline 232 & 0339 & 55.67517 & 32.95284 & \\
\hline 232 & 0525 & 55.50452 & 33.49902 & \\
\hline 232 & 0653 & 55.37593 & 33.97698 & \\
\hline 232 & 0714 & 55.34700 & 34.08950 & \\
\hline 232 & 0732 & 55.32345 & 34.16597 & 644 \\
\hline 232 & 0919 & 55.16727 & 34.73867 & \\
\hline 232 & 0957 & 55.13951 & 34.86032 & \\
\hline 232 & 1305 & 55.00515 & 35.41145 & \\
\hline 232 & 1335 & 54.95007 & 35.56577 & \\
\hline 232 & 2028 & 54.44653 & 37.08700 & \\
\hline 232 & 2301 & 54.21368 & 37.78762 & \\
\hline 233 & 0050 & 54.01300 & 38.30380 & \\
\hline 233 & 0151 & 53.90082 & 38.61642 & \\
\hline 233 & 0229 & 53.83395 & 38.79727 & \\
\hline 233 & 0417 & 53.66767 & 39.32900 & \\
\hline 233 & 0502 & 53.59883 & 39.52917 & \\
\hline 233 & 0604 & 53.52650 & 39.77550 & \\
\hline 233 & 0650 & 53.44967 & 40.00633 & \\
\hline 233 & 1122 & 52.97438 & 41.17592 & \\
\hline 233 & 1216 & 52.88085 & 41.42162 & \\
\hline 233 & 1311 & 52.78593 & 41.68168 & \\
\hline 233 & 1348 & 52.72997 & 41.84795 & \\
\hline 233 & 1534 & 52.55030 & 42.34230 & \\
\hline 233 & 2142 & 52.02162 & 43.65110 & \\
\hline 233 & 2238 & 51.93185 & 43.88240 & \\
\hline 234 & 0000 & 49.44278 & 49.25048 & \\
\hline 234 & 0106 & 51.70500 & 44.44164 & \\
\hline 234 & 0413 & 51.41883 & 45.18567 & \\
\hline 234 & 0439 & 51.37667 & 45.26633 & \\
\hline 234 & 0701 & 51.14067 & 45.75583 & \\
\hline 234 & 0808 & 51.01472 & 45.97807 & \\
\hline 234 & 0955 & 50.80985 & 46.35817 & \\
\hline 234 & 1059 & 50.68845 & 46.56553 & \\
\hline 234 & 1144 & 50.61105 & 46.75128 & \\
\hline 234 & 1240 & 50.51497 & 46.95382 & \\
\hline 234 & 1312 & 50.45560 & 47.07215 & \\
\hline 234 & 1424 & 50.33823 & 47.34165 & \\
\hline 234 & 1458 & 50.28283 & 47.46883 & \\
\hline 234 & 1610 & 50.16600 & 47.72867 & \\
\hline 234 & 1647 & 50.11370 & 47.84998 & \\
\hline 234 & 1720 & 50.06057 & 47.94490 & \\
\hline 234 & 1930 & 49.86522 & 48.34888 & \\
\hline 234 & 2104 & 49.72325 & 48.65190 & \\
\hline 234 & 2212 & 49.62015 & 48.86345 & \\
\hline 235 & 0002 & 49.44278 & 49.25048 & \\
\hline 235 & 0118 & 49.31655 & 49.50980 & \\
\hline 235 & 0150 & 49.26155 & 49.61173 & \\
\hline 235 & 0600 & 48.78428 & 50.38194 & \\
\hline 235 & 0730 & 48.62535 & 50.67238 & \\
\hline 235 & 0748 & 48.59307 & 50.72637 & \\
\hline 235 & 1034 & 48.32385 & 51.23337 & \\
\hline 235 & 1104 & 48.27488 & 51.32645 & \\
\hline 235 & 1314 & 48.05302 & 51.73962 & \\
\hline 235 & 1406 & 47.96520 & 51.90535 & \\
\hline
\end{tabular}




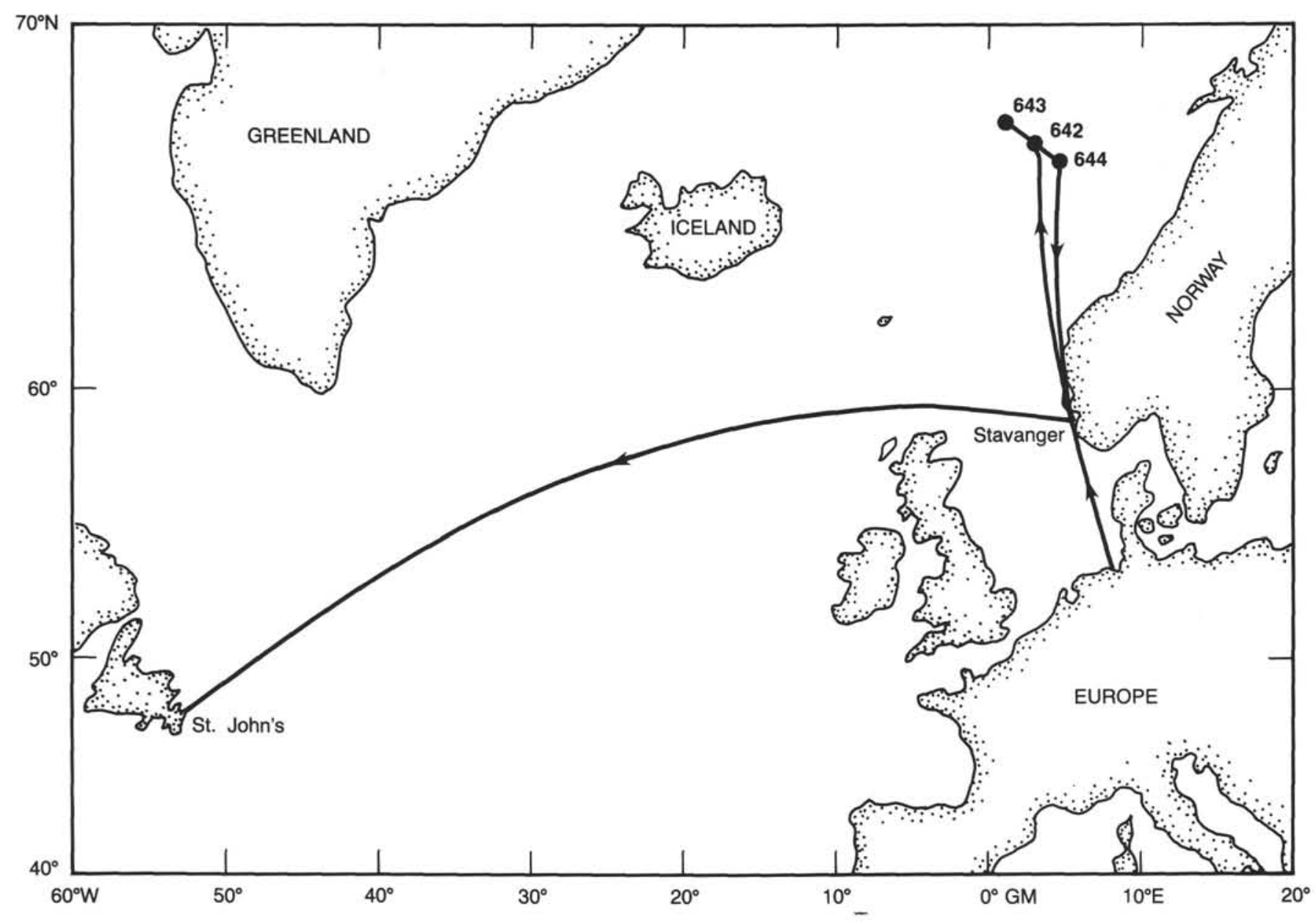

Figure 1. Leg 104 ship track. 


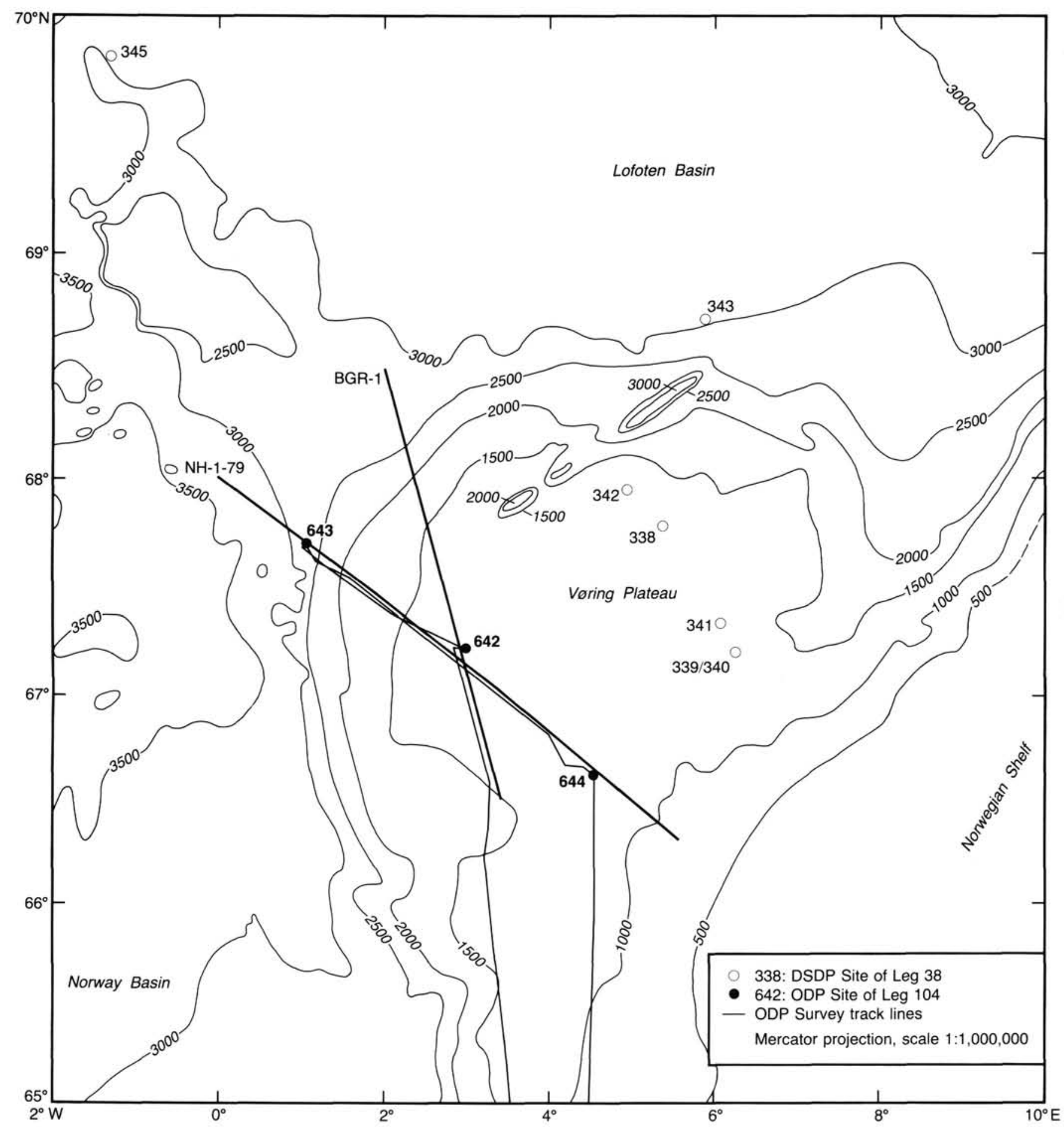

Figure 2. Positions of sites, and site survey profiles at the Vøring Plateau. Navigation for multichannel seismic lines BGR-1 and NH-1 is also shown. 

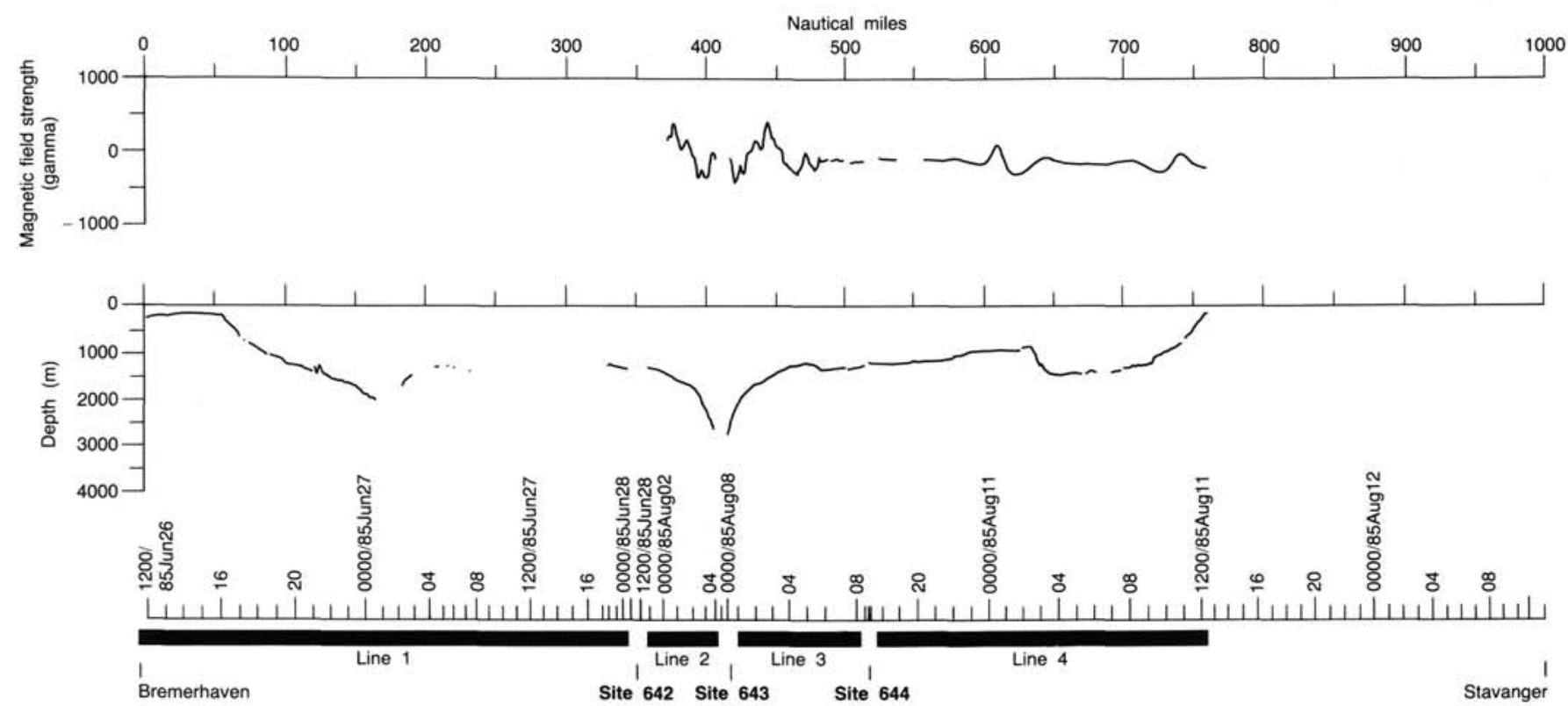

Figure 3. Magnetic and bathymetric profile records obtained during Leg 104A. Solid bars show the seismic reflection coverage during this portion of the leg.

Table 2. Real-time recording parameters for Leg 104 seismic data.

\begin{tabular}{|c|c|c|c|c|c|}
\hline & Line 1 & Line 2 & Line 3 & Line 4 & Line 5 \\
\hline Start at: & $\begin{array}{l}\text { Survey of } \\
\text { Site } 642\end{array}$ & Site 642 & Site 643 & Site 644 & Stavanger \\
\hline End at: & Site 642 & Site 643 & Site 644 & Stavanger & St. John's \\
\hline Source: & $\begin{array}{l}\text { Two } 80 \text {-in. }{ }^{3} \\
\text { Waterguns }\end{array}$ & $\begin{array}{l}\text { Two } 80 \text {-in. }{ }^{3} \\
\text { Waterguns }\end{array}$ & $\begin{array}{l}\text { Two } 80 \text {-in. }{ }^{3} \\
\text { Waterguns }\end{array}$ & $\begin{array}{l}\text { Two } 80 \text {-in. }{ }^{3} \\
\text { Waterguns }\end{array}$ & See text \\
\hline Streamer: & Port & Port & Port & Port & Port or Starboard \\
\hline \multicolumn{6}{|l|}{ EDO 1: } \\
\hline High Cut: & $190-200 \mathrm{~Hz}$ & $120-190 \mathrm{~Hz}$ & $180 \mathrm{~Hz}$ & $200 \mathrm{~Hz}$ & $200 \mathrm{~Hz}$ \\
\hline Low Cut: & $80-40 \mathrm{~Hz}$ & $20-40 \mathrm{~Hz}$ & $80 \mathrm{~Hz}$ & $80 \mathrm{~Hz}$ & $70-80 \mathrm{~Hz}$ \\
\hline \multicolumn{6}{|l|}{ Gain: } \\
\hline Amp: & Unknown & $20-30 \mathrm{db}$ & $80 \mathrm{db}$ & $80 \mathrm{db}$ & $80 \mathrm{db}$ \\
\hline Recorder: & Unknown & Full & Variable & Variable & Variable \\
\hline \multicolumn{6}{|l|}{ EDO 2: } \\
\hline High Cut: & $200 \mathrm{~Hz}$ & $120-200 \mathrm{~Hz}$ & $180 \mathrm{~Hz}$ & $200 \mathrm{~Hz}$ & $200 \mathrm{db}$ \\
\hline Low Cut: & $40-60 \mathrm{~Hz}$ & $20-40 \mathrm{~Hz}$ & $80 \mathrm{~Hz}$ & $80 \mathrm{~Hz}$ & $80-90 \mathrm{~Hz}$ \\
\hline \multicolumn{6}{|l|}{ Gain: } \\
\hline Amp: & Unknown & $20-30 \mathrm{db}$ & $80 \mathrm{db}$ & $70-90 \mathrm{db}$ & $80 \mathrm{db}$ \\
\hline Recorder: & Unknown & Full & Variable & Variable & Variable \\
\hline
\end{tabular}

Table 3. Processing parameters for Leg 104 seismic data.

\begin{tabular}{llll}
\hline & Line 1 & Line 4 & Line 5 \\
\hline Data Window: & Variable & Variable & Variable \\
Plot start time: & Variable & Variable & Variable \\
Plot stop time: & Variable & Variable & Variable \\
Plot time scale: & 6.25 in./s & $4.00 \mathrm{in.} / \mathrm{s}$ & $4.00 \mathrm{in.} / \mathrm{s}$ \\
Zero-phase & & & \\
band-pass filter: & & & \\
$\quad$ High cut $(\mathrm{Hz}):$ & 250 & 250 & 250 \\
$\quad$ Low cut $(\mathrm{Hz}):$ & 20 & 20 & 20 \\
\hline
\end{tabular}



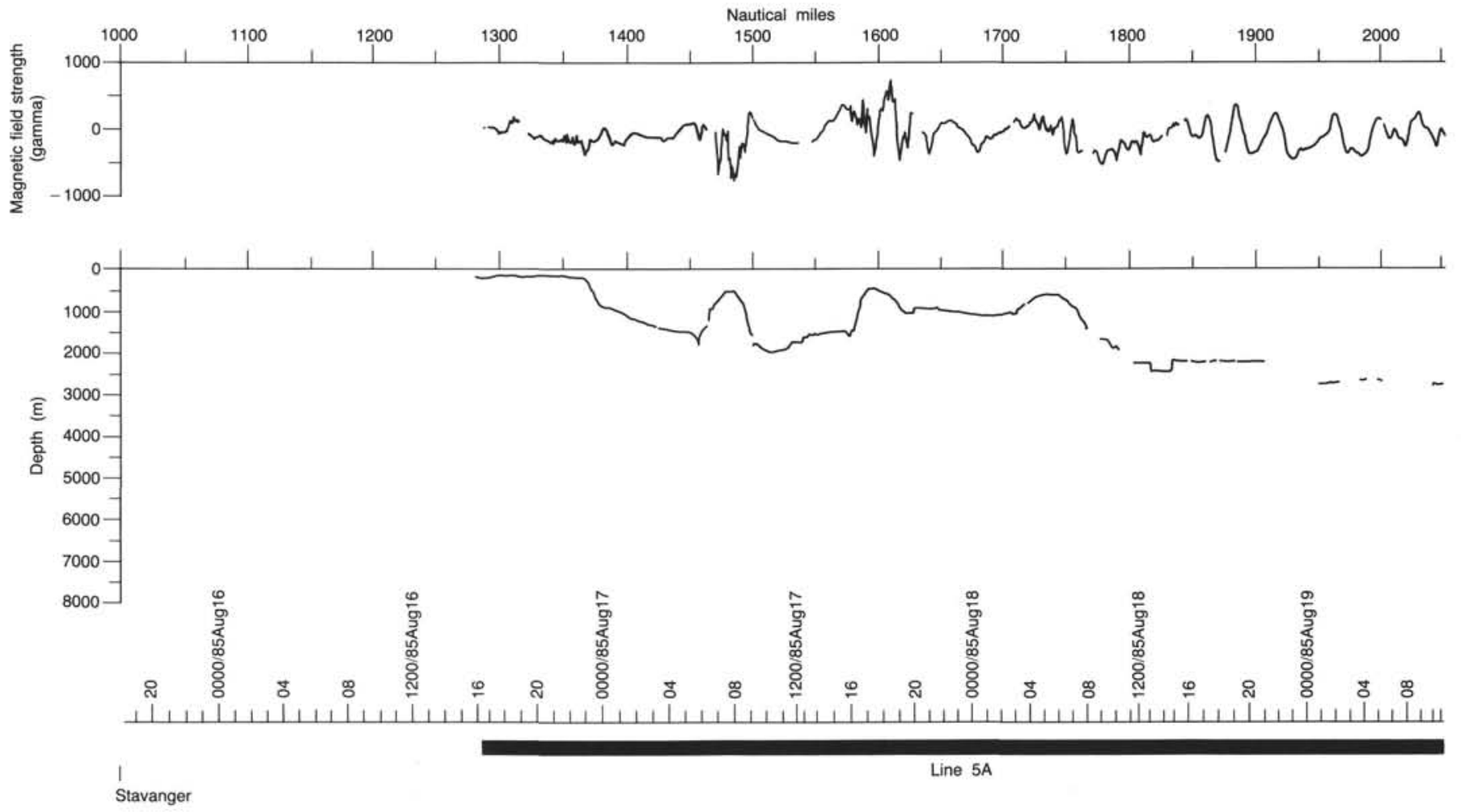

Figure 4. Magnetic and bathymetric profile records obtained during Leg 104B. Solid bars show the seismic-reflection coverage during this portion of the leg.
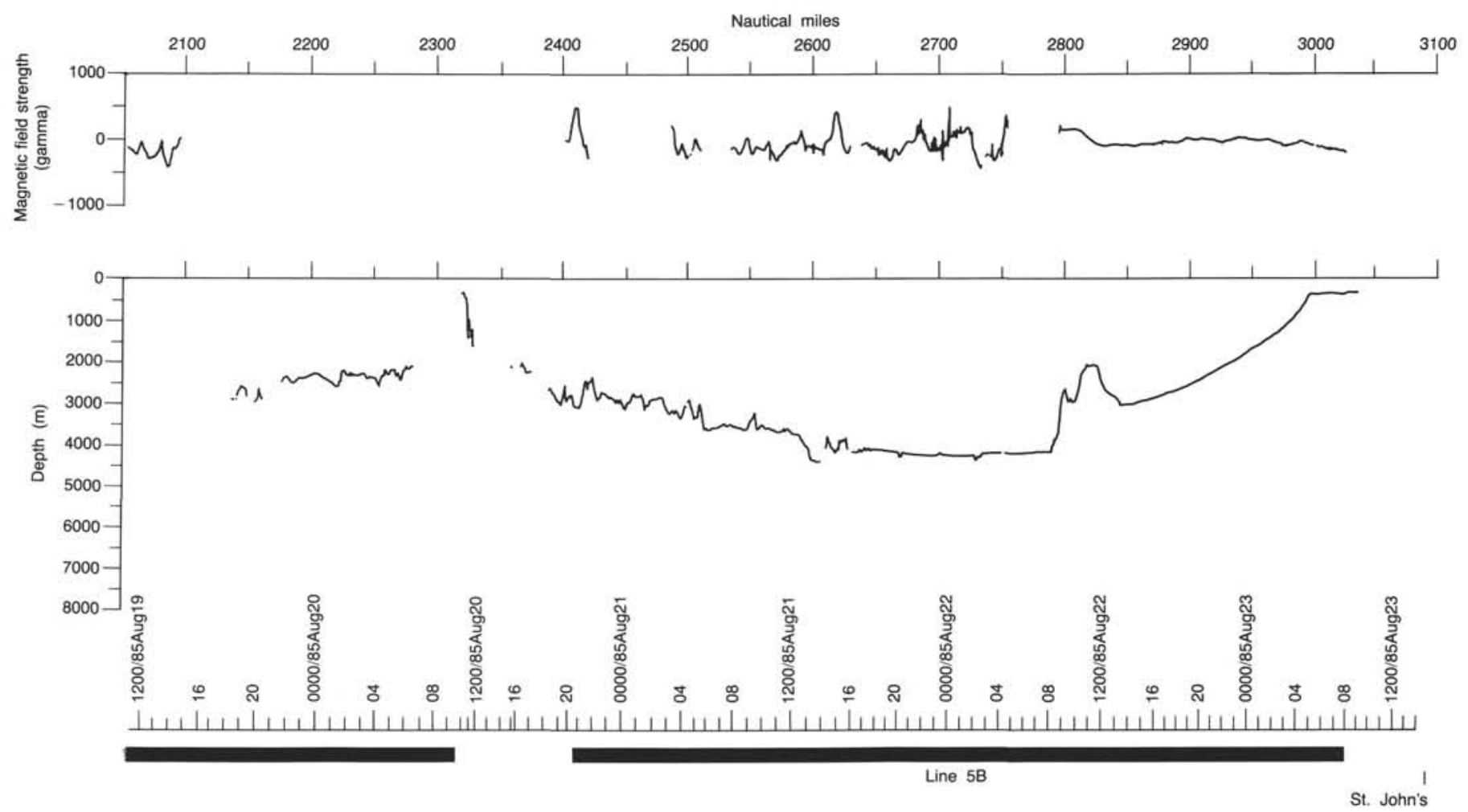

Figure 4 (continued). 